\title{
The Tumor Boards - is this strategy worth for developing countries?
}

\author{
Antonio Cassio de Assis Pellizzon, MD, PhD \\ AC Camargo Cancer Center, Sao Paulo, Brazil \\ E-mail: cassiopellizzon@aol.com
}

J Contemp Brachytherapy 2018; 10, 3: 191-192

DOI: https://doi.org/10.5114/jcb.2018.76958

Cancer treatment is becoming more complex and has a potential need of having physicians of various specialties participating in the treatment planning. In general, as a consequence of a confirmation of cancer diagnosis, patients are sent to specialists who examine them generally and perform diagnostic procedures in order to stage the disease and define accurate steps of treatment. These steps involve different oncologic medical specialists such as surgeons, radiation and clinical oncologists, apart from other supporting teams. This multidisciplinary management performed sequentially it's a time consuming approach that can create anxiety in patients and family members, factors that cannot be overlooked. In many situations of cancer treatment, patients can consider different management options, in which more time is spent when attending different clinics. In fact, usually more than one specialty involved in the treatment will be necessary to take the final decision about the treatment scheme. To reduce the time gap between diagnosis and starting of treatment, the introduction of Tumor Boards (TBs) through multidisciplinary clinics or meetings, which are formal with the presence of key specialists to discuss the diagnosis and management of cancer patients, can be a helpful tool [1]. By definition, the TBs tend to be held on weekly, regularly basis. Besides its educational functions, the attendance of radiation oncologists, medical oncologists, oncologic surgeons, radiologists, pathologists, and other specialists, depending on the type and origin of cancer, gives a unique chance to review each and every case individually [2]. Furthermore, the advent of the Internet has led to an increased number of patients with better knowledge and higher cancer awareness who seek a multidisciplinary clinic for second opinion. It is important to note that TBs are not designed to be a form of second opinion but in most cases, they are used for this purpose, especially because expert's opinions are based on case exams and literature reviews to present an evidence-based recommendation.

There are few studies that investigated TBs recommendations and their impact on the oncologic outcomes. One survey of Oregon hospitals found that TBs recommendations were generally implemented into patient care, and played a strategic role in the treatment planning for cancer patients [3]. Another single-institutional study of the Johns Hopkins Hospital, published in 2008, evaluated the impact of multidisciplinary clinic on the pre-management of pancreatic cancer. They found that changes in therapeutic recommendations occurred in up to $25 \%$ of the patients [4]. Another study from the University of Michigan Comprehensive Cancer Center, in which Ann Arbor is reviewing records of patients referred to a multidisciplinary breast cancer clinic over a 1-year period. The author concluded that changes in management stemmed from differences in mammographic and pathologic interpretation, plus the evaluation by medical, radiation oncologists, and surgical breast specialists. It was also discovered that the meetings changed surgical recommendations for more than $50 \%$ of patients with breast cancer [5]. Kesson et al. evaluated the effect of multidisciplinary care on survival of 13,722 women treated for breast cancer at NHS hospitals in western Scotland, UK, observing that the introduction of TBs in clinical practice was associated with improved survival and reduced variation in survival among hospitals [6].

On the other hand, a review including 18 original studies comparing the introduction of a TB and its relation to patient survival showed that 12 of these studies reported statistically significant association between presence of TBs and patient survival, but the final conclusion was that due to methodological limitations, the review was unable to assert a causal relationship between TBs and patient survival [7]. Another study of Veterans Affairs Medical Centers found no associations between TBs and either patient survival or process measures [8].

The ASCO (American Society of Clinical Oncology) in the 2014 ASCO Educational Book described two versions of tumor boards: one, where all cases are discussed briefly and another, where only specific and complex cases are discussed in depth. This publication also suggests variants of TBs organized at selected cancer centers, where basic molecular and microarray translational research are applied in the so-called 'molecular tumor boards'. The ASCO publication also emphasized that patients with more complex needs that are referred to oncologic centers should have the chance to enter oncological clinical trials in order to strengthen oncology care even more [9].

In conclusion, the introduction of TBs, despite no definitive assumption of its impact on survival, could improve the time spent on cancer staging and treatment planning, limiting the number of clinics to be attended 
by patients. In resource limiting or continental dimension countries, where there is a lack of cancer centers and specialized facilities, the time and resources consumption savings could influence founds saving of public and private health systems. This could result in increasing number of reference centers for cancer treatment.

\section{Disclosure}

The author reports no conflict of interest.

\section{References}

1. National Cancer Institute. Definition of Tumor Board Review. Available at: http:// www. cancer.gov/dictionary?cdrid322893. Accessed: 11 December 2017.

2. Gatcliffe TA, Coleman RL. Tumor board: More than treatment planning - A 1-year prospective survey. J Cancer Educ 2008; 23: 235-237.

3. Petty JK, Vetto JT. Beyond doughnuts: Tumor board recommendations influence patient care. J Cancer Educ 2002; 17: 97-100.

4. Pawlik TM, Laheru D, Hruban RH et al. Johns Hopkins Multidisciplinary Pancreas Clinic Team. Evaluating the impact of a single-day multidisciplinary clinic on the management of pancreatic cancer. Ann Surg Oncol 2008; 15: 2081-2088.

5. Newman EA, Guest AB, Helvie MA et al. Changes in surgical management resulting from case review at a breast cancer multidisciplinary tumor board. Cancer 2006; 107: 2346-2351.

6. Kesson EM, Allardice GM, George WD et al. Effects of multidisciplinary team working on breast cancer survival: retrospective, comparative, interventional cohort study of 13,722 women. BMJ 2012; 26: e2718.

7. Hong NJ, Wright FC, Gagliardi AR et al. Examining the potential relationship between multidisciplinary cancer care and patient survival: an international literature review. J Surg Oncol 2010; 102: 125-134.

8. Blayney DW. Tumor boards (team huddles) aren't enough to reach the goal. J Natl Cancer Inst 2013; 105: 82-84.

9. https://meetinglibrary.asco.org/record/89313/edbook\#fulltext. Accessed: 12 December 2017. 